This item was submitted to Loughborough's Research Repository by the author.

Items in Figshare are protected by copyright, with all rights reserved, unless otherwise indicated.

\title{
Acute high-intensity interval running reduces postprandial lipemia in boys
}

PLEASE CITE THE PUBLISHED VERSION

http://dx.doi.org/10.1249/MSS.0b013e31828452c1

\section{PUBLISHER}

(c) The American College of Sports Medicine

\section{VERSION}

AM (Accepted Manuscript)

\section{LICENCE}

CC BY-NC-ND 4.0

\section{REPOSITORY RECORD}

Thackray, Alice E., Laura A. Barrett, and Keith Tolfrey. 2019. "Acute High-intensity Interval Running Reduces Postprandial Lipemia in Boys”. figshare. https://hdl.handle.net/2134/12627. 
This item was submitted to Loughborough's Institutional Repository (https://dspace.lboro.ac.uk/) by the author and is made available under the following Creative Commons Licence conditions.

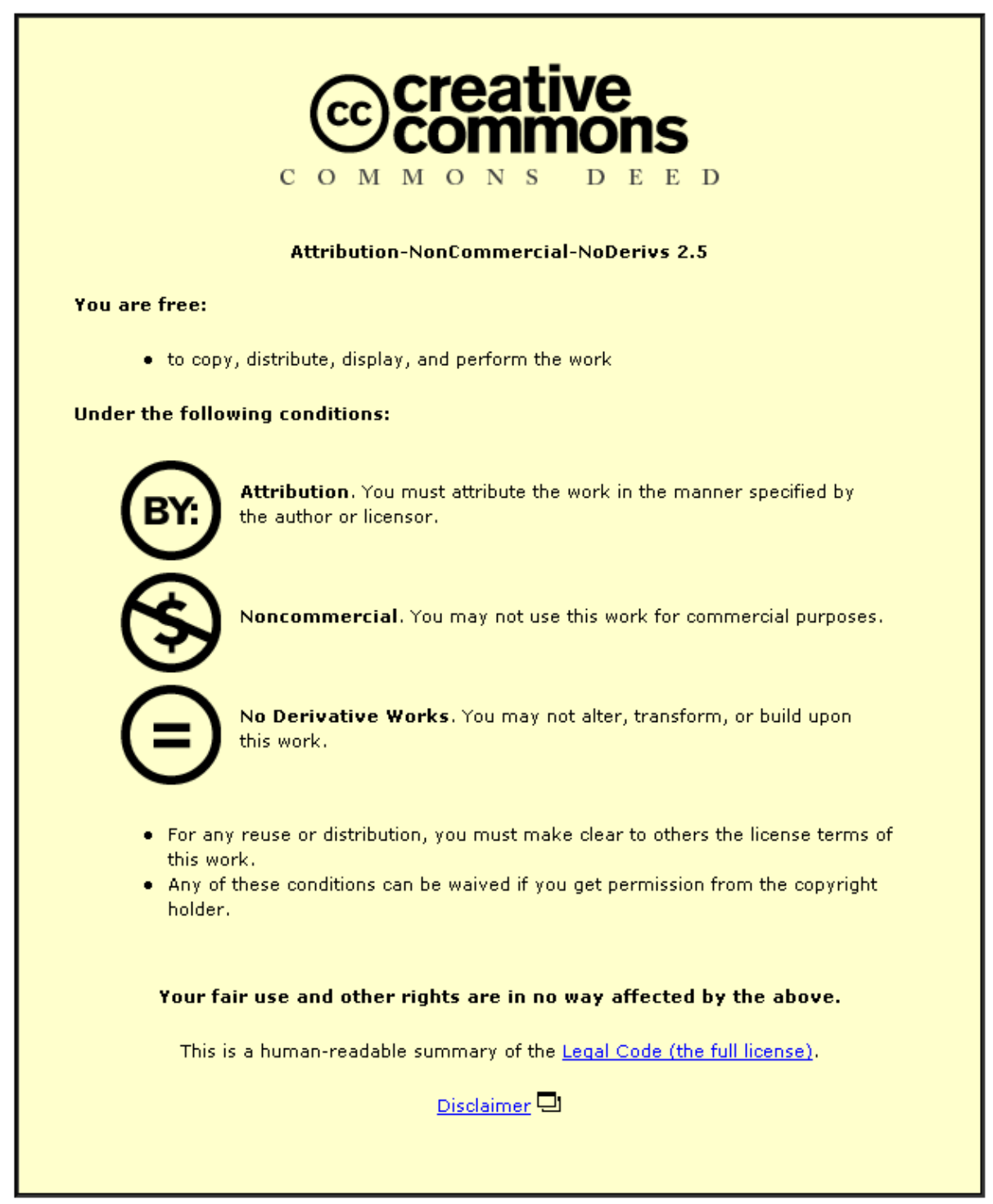

For the full text of this licence, please go to: http://creativecommons.org/licenses/by-nc-nd/2.5/ 


\title{
Acute High-Intensity Interval Running Reduces Postprandial Lipemia in Boys
}

\author{
Alice E. Thackray, Laura A. Barrett, and Keith Tolfrey \\ School of Sport, Exercise and Health Sciences, Loughborough University, \\ Loughborough, United Kingdom
}

Accepted for Publication: 20 December 2012

Medicine \& Science in Sports \& Exercise ${ }_{\circledast}$ Published ahead of Print contains articles in unedited manuscript form that have been peer reviewed and accepted for publication. This manuscript will undergo copyediting, page composition, and review of the resulting proof before it is published in its final form. Please note that during the production process errors may be discovered that could affect the content. 


\section{Acute High-Intensity Interval Running Reduces}

\section{Postprandial Lipemia in Boys}

Alice E. Thackray ${ }^{1}$, Laura A. Barrett ${ }^{1}$ and Keith Tolfrey ${ }^{1}$

${ }^{1}$ School of Sport, Exercise and Health Sciences, Loughborough University, Loughborough, LE11 3TU, UK

\section{Corresponding author:}

Dr. Keith Tolfrey

School of Sport, Exercise and Health Sciences

Loughborough University

Loughborough

LE11 3TU

UK

Phone +44(0)1509 226355

Fax +44(0)1509226301

Email: k.tolfrey@lboro.ac.uk 
Conflicts of interest and Source of Funding: The authors declare no conflict of interest. No funding was received for this research, other than that available internally through Loughborough University.

Running title: High-intensity running and lipemia 


\section{Abstract}

Introduction: Acute moderate-intensity exercise reduces postprandial lipemia in boys.

However, the effect of high-intensity exercise has not been investigated. This study examined the effect of low-volume, high-intensity interval running (HIIR) on postprandial plasma triacylglycerol concentrations [TAG]. Methods: Fifteen healthy, active boys (means(SD): age $11.8(0.4)$ years; body mass $42.8(8.0) \mathrm{kg}$; peak oxygen uptake $\left.\left(\dot{\mathrm{V}}{ }_{2}\right) 55(6) \mathrm{mL} \cdot \mathrm{kg}^{-1} \cdot \mathrm{min}^{-1}\right)$ completed two, 2-day trials in a counter-balanced, cross-over design separated by 14 days. On day 1 , participants rested $(\mathrm{CON})$ or completed $10 \times 1$ min running intervals at $100 \%$ maximal aerobic speed, determined from an incremental peak $\dot{\mathrm{VO}}_{2}$ test, with 1 min recovery between intervals (HIIR). On day 2, capillary blood samples were taken in the fasted state and at pre-determined intervals throughout the $6.5 \mathrm{~h}$ postprandial period while participants rested. A standardised breakfast was consumed at 08:00 immediately after the fasting sample and a standardised lunch meal at 12:00. Results: Differences in fasting plasma [TAG] were small to moderate $(95 \%$ confidence interval $(95 \% \mathrm{CI})-0.11$ to 0.01 , Effect size $(\mathrm{ES})=0.40)$. Postprandial [TAG] was lower during HIIR compared with CON (95\% CI -0.19 to -0.02, ES $=0.58)$. The total area under the $[\mathrm{TAG}]$ versus time curve was lower following HIIR compared with CON (5.2(1.1) vs. 5.8(1.5) $\mathrm{mmol} \cdot \mathrm{L}^{-1} 6.5 \mathrm{~h}$; $95 \% \mathrm{CI}-1.18$ to -0.12 , ES = 0.50). Conclusion: This is the first study to show that low volume, high-intensity interval running attenuates postprandial [TAG] in healthy, active 11 to 12 year old boys.

Keywords: PPL, TAG, exercise intensity, young people 


\section{INTRODUCTION}

Elevated plasma triacylglycerol concentrations [TAG], especially in the postprandial period, have been implicated in the development of atherosclerosis (42). Impaired clearance of postprandial [TAG] is associated independently with an increased risk of future cardiovascular diseases $(1,33)$, which remain the leading cause of death in the UK (36). Most people typically spend the majority of the daytime in a postprandial state; therefore, repeated daily exposure to elevated postprandial [TAG] promotes the development of an atherogenic lipid profile of small, dense low-density lipoprotein and low concentrations of high-density lipoprotein (7). Considering the process of atherosclerosis is initiated during childhood, lifestyle interventions that moderate postprandial lipemia by improving TAG metabolism may slow atherogenic progression, even during childhood and adolescence (32).

Acute moderate-intensity exercise ( $30 \mathrm{~min}$ to $3 \mathrm{~h}$ in duration) performed up to $16 \mathrm{~h}$ before a standardised meal reduces postprandial lipemia in adults (34), although this effect is short-lived (19) and may be dependent on the exercise-induced energy expenditure (16). Similar postprandial studies with boys have also reported acute reductions in postprandial lipemia following a single moderate-intensity exercise session $(2,29,39,41)$. Barrett et al. (2) suggested the larger reduction in postprandial [TAG] after intermittent-games activity compared with continuous exercise may reflect the higher exercise energy expenditure (EE). However, the evidence is limited by the between-measures design and the inability to estimate the exercise EE during the intermittent-games activity (2). Subsequent studies suggest a dose-dependent response is not supported in healthy young people $(39,41)$.

Recent findings have suggested that low volume, high-intensity interval training results in similar physiological and metabolic adaptations as higher volume, moderate to vigorous intensity continuous training $(21,28)$. Furthermore, this type of training may also 
improve insulin sensitivity and glycaemic control (28). Acute intermittent high-intensity exercise has also been shown to attenuate the postprandial lipaemic response in healthy adult males $(11,13,14)$. However, it is not known whether a single session of low volume, highintensity exercise attenuates the plasma TAG response to standardised meals in boys. Current international guidelines recommend that children and adolescents accumulate at least 60 min of moderate daily physical activity to promote and maintain health $(9,23)$. Considering that many children and adolescents fail to meet these guidelines (35), low volume, high-intensity exercise may represent a viable alternative to help improve health and increase physical activity participation from a young age (4).

Therefore, the aim of the present study was to examine the effect of a single bout of low volume, high-intensity interval running (HIIR) on postprandial lipemia in healthy, active 11 to 12 year old boys. It was hypothesised that acute, HIIR would effectively reduce postprandial $[\mathrm{TAG}]$ compared with a resting control despite the relatively low exercise energy expenditure.

\section{METHODS}

\section{Participants}

After approval from the University Ethical Advisory Committee, 16 boys aged 11.3 to 12.9 years volunteered to participate in this study. Results are presented for 15 boys as one boy dropped out due to illness. Written assent was obtained from each participant and written informed consent by a parent or guardian. A health screen questionnaire revealed all participants were in good general health, had no history of medical conditions that may compromise exercise participation and were not taking any medications or dietary 
supplements known to influence lipid or carbohydrate metabolism. All participants indicated they were actively participating in sport, but not specifically accustomed to high-intensity running. Physical and physiological characteristics of participants are presented in Table 1.

\section{Anthropometry and physical maturation}

All anthropometric measurements were conducted with participants wearing shorts, T-shirt and socks. Stature was measured to the nearest $0.01 \mathrm{~m}$ using a fixed stadiometer (Holtain, Crosswell, UK), and body mass was quantified to the nearest $0.1 \mathrm{~kg}$ using a balance-beam scale (Avery, Birmingham, UK). Body mass index (BMI) was calculated as body mass (kg) divided by stature (m) squared. Triceps and subscapular skinfold thickness were measured to the nearest $0.2 \mathrm{~mm}$ on the right-hand side of the body using Harpenden callipers (John Bull, St. Albans, UK). The median of three measurements at each site was used to estimate percent body fat $(\% \mathrm{BF})$ using the maturation, race and sex-specific equations developed by Slaughter et al. (37). Lean body mass (LBM) was estimated:

$$
\operatorname{LBM}(\mathrm{kg})=\text { body mass }(\mathrm{kg}) \times[1-(\% \mathrm{BF} / 100)]
$$

Level of physical maturity was estimated through self-assessment of secondary sex characteristics. Participants were provided with drawings depicting the five stages of genital and pubic hair development (38), ranging from 1 indicating pre-pubescence to 5 indicating full sexual maturity, and were required to identify the stage reflecting their current level of sexual development. 


\section{Preliminary exercise measurements}

During the first visit to the laboratory, participants were familiarised with walking and running on the treadmill (Technogym Runrace, Gambettola, Italy) before completing an incremental speed-based treadmill protocol. This protocol was designed to determine peak oxygen uptake $\left(\mathrm{V}_{2}\right)$ and maximal aerobic speed (MAS), defined as the running speed eliciting the highest $\dot{\mathrm{V}}_{2}$ during an incremental test (27). The protocol started at an initial speed of $6.0 \mathrm{~km} \cdot \mathrm{h}^{-1}$ with $0.5 \mathrm{~km} \cdot \mathrm{h}^{-1}$ increments every $30 \mathrm{~s}$ until volitional exhaustion, with the treadmill gradient set at $1 \%$ throughout (25). Heart rate was monitored continuously via short-range telemetry (PE4000, Polar-Electro, Kempele, Finland) and ratings of perceived exertion (RPE) were recorded in the last $10 \mathrm{~s}$ of each $30 \mathrm{~s}$ stage (3). Expired air samples were monitored continuously using an online breath-by-breath gas analysis system (Metalyzer 3B, Cortex, Leipzig, Germany). The analyser was calibrated before each test using a bottled gas mixture containing 5.01\% $\mathrm{CO}_{2}, \quad 16.98 \% \mathrm{O}_{2}$ and balance $\mathrm{N}_{2}$ (Cranlea and Company, Birmingham, UK) and a 3.0 L syringe (Hans Rudolf, Shawnee, USA). Participants wore an appropriate size facemask (Hans Rudolf, Shawnee, USA) which was checked for leaks and connected to the online system via a flowmeter before the exercise began. All participants satisfied at least two of the following criteria to confirm attainment of maximal effort during the peak $\dot{\mathrm{V}} \mathrm{O}_{2}$ test: a plateau in $\dot{\mathrm{VO}}_{2}(\leq 3 \%)$ with an increase in treadmill speed; a peak heart rate $\geq 95 \%$ of age-predicted maximum (220-chronological age); and a respiratory exchange ratio $\geq 1.10$. An average of the breath-by-breath $\dot{\mathrm{V}} \mathrm{O}_{2}$ data was taken every $10 \mathrm{~s}$, and peak $\dot{\mathrm{V} O}$ 2 was defined as the highest $30 \mathrm{~s}$ rolling average; the treadmill speed corresponding to peak $\dot{\mathrm{V}} \mathrm{O}_{2}$ was recorded as MAS. 


\section{Experimental design}

The experimental design was similar to previous studies with boys $(2,39,41)$. Using a within-measures, counter-balanced, cross-over design, participants completed two, 2-day experimental conditions: a resting control condition $(\mathrm{CON})$ and a high-intensity interval running condition (HIIR). The conditions were separated by a standardised period of 14 days. The study design is presented schematically in Figure 1.

\section{Day 1}

On day 1 at 15:30 h, participants arrived at the laboratory and all measures were completed by $16: 30 \mathrm{~h}$. Body mass was recorded at the start of each experimental condition to standardise the test meals provided on day 2. During HIIR, participants completed a 5 min warm-up at $60 \%$ MAS, followed immediately by the acute high-intensity running intervals and concluding with a $3 \mathrm{~min}$ cool-down at $40 \%$ MAS. The high-intensity exercise session involved $10 \times 1$ min treadmill runs at 100\% MAS with 1 min active recovery between each interval. Several recent studies adopting a low-volume, high-intensity exercise session (10 x 1 min high-intensity cycle sprints, 1 min recovery) reported that this protocol was well tolerated in patients with type 2 diabetes mellitus $(21,28)$. Following initial pilot work with 12 to 14 year old adolescents, this pattern of exercise on the treadmill was deemed suitable for this population. During the active recovery period, participants dismounted the treadmill and were encouraged to pace around the lab to avoid venous pooling and feeling light headed. Heart rate was monitored continuously and the participants provided RPE in the last $10 \mathrm{~s}$ of each interval as described previously. During CON, participants rested in the laboratory to match the duration of HIIR. 
Day 2

On day 2 at $\sim 07: 45 \mathrm{~h}$, participants arrived at the laboratory following a $12 \mathrm{~h}$ overnight fast and provided a fasting capillary blood sample after 10 min seated rest. Participants then consumed a standardised test breakfast meal within 15 min, marking the start of the postprandial period $(08: 00 \mathrm{~h})$. Subsequent capillary blood samples were taken at $0.5,1,3$, 4.5, 5 and $6.5 \mathrm{~h}$ in the postprandial period, with participants consuming a standardised test lunch meal within $20 \mathrm{~min}$ at $4 \mathrm{~h}$ (Figure 1). To reduce the impact of any physical activity or postural variations on the postprandial measures, participants remained seated throughout the day and were able to read, watch DVD films and play non-active computer games. Participants consumed water ad libitum in the postprandial period of the first condition and the ingested volume was replicated in the subsequent condition.

\section{Standardisation of diet and physical activity}

Participants recorded their dietary intake and all physical activity categorised according to intensity level during the $48 \mathrm{~h}$ period before day 2 of the first experimental condition. They were asked to minimise and record their physical activity in a diary during this period. The boys then replicated this diet and physical activity pattern before the second experimental condition - this was confirmed verbally by the lead investigator. The overnight fasting period was standardised by asking participants to consume a small carbohydrate-rich cereal snack bar at 19:45 h on Day 1 of each experimental condition. The macronutrient content of the cereal snack bar was $1.2 \mathrm{~g}$ fat, $16.1 \mathrm{~g}$ carbohydrate and $1.0 \mathrm{~g}$ protein, which provided $334 \mathrm{~kJ}$ energy. After 20:00 h the participants were asked to only drink plain water before arriving at the laboratory on day 2. Two-day food records were analysed using dietary analysis software (CompEat Pro Version 5.8.0, Nutrition Systems, Banbury, UK). 


\section{Test meals}

The test breakfast consisted of croissants, chocolate spread, whole milk, double cream and milkshake powder. The meal quantity was prescribed relative to body mass and provided 1.5 $\mathrm{g}$ fat $(60 \%$ of total energy), $1.8 \mathrm{~g}$ carbohydrate $(33 \%), 0.4 \mathrm{~g}$ protein $(7 \%)$ and $93 \mathrm{~kJ}$ energy per kilogram body mass. The test lunch consisted of white bread, mild cheddar cheese, butter, potato crisps, whole milk and milkshake powder and provided $1.1 \mathrm{~g}$ fat $(50 \%), 1.9 \mathrm{~g}$ carbohydrate $(38 \%), 0.6 \mathrm{~g}$ protein $(12 \%)$ and $86 \mathrm{~kJ}$ energy per kilogram body mass. To ensure consistency across participants and experimental conditions, participants consumed either chocolate or strawberry flavour milkshake powder on both visits. The time taken to consume each meal during the first experimental condition was recorded and replicated in the remaining condition.

\section{Analytical methods}

Capillary blood samples were taken after the hand was pre-warmed in water heated to $40^{\circ} \mathrm{C}$ for $5 \mathrm{~min}$. The fingertip was pierced (Unistick 3 Extra, Owen Mumford, UK) and $600 \mu \mathrm{L}$ of whole capillary blood was collected into potassium-EDTA coated Microvette CB 300 tubes (Sarstedt Ltd, Leicester, UK). The whole blood samples were immediately centrifuged at 12,800 g for 15 min (Eppendorf 5415c, Hamburg, Germany). An automatic pipette was used to dispense $200 \mu \mathrm{L}$ of plasma into a $0.5 \mathrm{ml}$ Eppendorf tube (Fisher Scientific Ltd, Loughborough, UK). The plasma samples were stored at $-80^{\circ} \mathrm{C}$ for up to two months before subsequent analyses. Plasma [TAG] and glucose concentration ([glucose]) were analysed by enzymatic, colorimetric methods (Randox Laboratories Ltd, Crumlin, UK) using a benchtop analyser (Pentra 400, HORIBA ABX Diagnostics, Montpellier, France). Plasma insulin concentration ([insulin]) was quantified using a commercially available enzyme-linked 
immunoassay (Mercodia Insulin ELISA, Mercodia AB, Uppsala, Sweden). The within-batch coefficients of variation for [TAG], [glucose] and [insulin] were 1.0, 0.4 and $4.1 \%$ respectively. Haemoglobin concentration and haematocrit were also quantified in duplicate in the fasting and final postprandial samples to estimate the acute change in plasma volume (10). Haemoglobin concentration was assessed using the cyanmethemoglobin method; $20 \mu \mathrm{L}$ whole blood was added to $5 \mathrm{~mL}$ Drabkin's solution and the absorbance was quantified photometrically at a wavelength of $546 \mathrm{~nm}$ (Cecil CE1011, Cecil instruments, Cambridge, England). Haematocrit was quantified using a microhaematocrit centrifuge and reader (Haematospin 1300 Microcentrifuge, Hawksley and Sons Ltd, Sussex, UK).

\section{Statistical analyses}

Data were analysed using the IBM SPSS Statistics software for Windows version 19 (IBM Corporation, New York, USA). All results are expressed as mean (SD). Descriptive statistics illustrating the physical and physiological characteristics of all participants and exercise responses were calculated (Table 1). Normality of the data was checked by Shapiro Wilk tests. Homogeneity of variances was confirmed by Mauchly's test of sphericity, and a Greenhouse-Geisser correction was applied to the degrees of freedom if the sphericity assumption was violated. The trapezium rule was used to calculate the total area under the plasma concentration versus time curves for TAG (TAUC-TAG), glucose (TAUC-glucose) and insulin (TAUC-insulin) (Table 2). The incremental areas under the plasma concentration versus time curves for TAG (iAUC-TAG), glucose (iAUC-glucose) and insulin (iAUCinsulin) were calculated using the same method after correcting for fasting concentrations. Energy and macronutrient intake along with TAUC and iAUC responses, fasting [TAG], [glucose] and [insulin] and estimated changes in plasma volume were compared between 
each experimental condition using separate one-way within-measures ANOVA. Differences in [TAG], [glucose] and [insulin] over the total $6.5 \mathrm{~h}$ postprandial period were examined using separate $2 \times 7$ (condition by time) within-measures ANOVA. A 2 × 3 (condition by time) within-measures ANOVA was conducted, and followed up with simple planned contrasts, to identify differences in TAUC-TAG between CON and HIIR over sub-sections of the total postprandial period ( 0 to $1 \mathrm{~h}, 1$ to $4.5 \mathrm{~h}$ and 4.5 to $6.5 \mathrm{~h}$ ). The $95 \%$ confidence intervals $(95 \% \mathrm{CI})$ for mean absolute pairwise differences between experimental conditions were calculated using the t-distribution and degrees of freedom $(n-1)$ and absolute standardised effect sizes (ES) are included to supplement important findings (12). In the absence of a clinical anchor, an ES of 0.2 was considered the minimum important difference for all outcome measures, 0.5 moderate and 0.8 large (6). Bivariate correlations identifying possible determinants of exercise-induced changes in TAUC-TAG were quantified using linear regression. Interpretation of the data will be based on the $95 \%$ CI and ES rather than more traditional dichotomous hypothesis testing.

\section{RESULTS}

\section{Dietary intake}

Average energy intake was similar during the $48 \mathrm{~h}$ before day 2 of CON and HIIR (8.1(1.6)

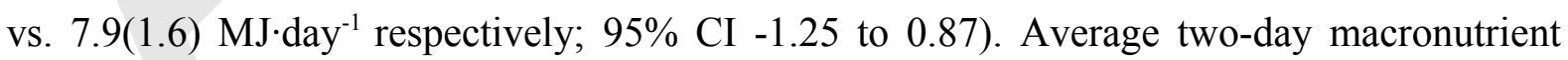
intake did not differ between CON and HIIR for protein $\left(69.0(15.7)\right.$ vs. 68.8(14.3) $\mathrm{g} \cdot \mathrm{day}^{-1}$; 95\% CI -8.4 to 8.1$)$, carbohydrate $\left(270.0(48.4)\right.$ vs. $253.1(55.4) \mathrm{g} \cdot \mathrm{day}^{-1} ; 95 \% \mathrm{CI}-55.0$ to 21.2$)$ or fat $\left(64.1(20.8)\right.$ vs. $66.2(20.1) \mathrm{g} \cdot \mathrm{day}^{-1} ; 95 \% \mathrm{CI}-7.6$ to 11.7$)$ respectively. 


\section{Responses to high-intensity interval running}

The high-intensity interval running session was well tolerated by all participants and was performed at an average MAS of $12.5(1.6) \mathrm{km} \cdot \mathrm{h}^{-1}$. Mean heart rate increased progressively from interval 1 to interval 10 (interval 1: 184(8) vs. interval 10: 194(8) beats $\cdot \mathrm{min}^{-1} ; 95 \%$ CI 7 to $13, \mathrm{ES}=1.29)$, which corresponded to $92(3) \%$ and $97(2) \%$ peak heart rate respectively (95\% CI 4 to $6, \mathrm{ES}=1.88)$. Mean RPE during interval 1 was 10(3) (between very light and fairly light on the scale), but increased to 19(1) at the end of interval 10 (very, very hard) $(95 \%$ CI 7 to $11, \mathrm{ES}=3.97)$.

Plasma volume changes and fasting [TAG], [glucose] and [insulin]

Average changes in plasma volume between the fasting and $6.5 \mathrm{~h}$ postprandial samples were small and did not differ significantly between the two conditions (CON 0.46\%, HIIR 0.85\%; 95\% CI -3.77 to 4.57$)$. Therefore, the raw plasma [TAG], [glucose] and [insulin] were used in all statistical analyses without adjustment. The fasting plasma [TAG], [glucose] and [insulin] for each condition are shown in Table 2. Differences in fasting plasma [TAG] were small to moderate $(95 \% \mathrm{CI}-0.11$ to $0.01, \mathrm{ES}=0.40)$, with a slightly lower fasting [TAG] evident after HIIR. There were no differences in fasting plasma [glucose] (95\% CI -0.33 to 0.19$)$ or [insulin] (95\% CI -19.0 to 16.9) between CON and HIIR.

Plasma [TAG], [glucose] and [insulin] in the postprandial period

Plasma $[\mathrm{TAG}]$ responses over the postprandial period for the experimental conditions are shown in Figure 2. Postprandial plasma [TAG] was lower during HIIR compared with CON (main effect condition $95 \% \mathrm{CI}-0.19$ to -0.02 , $\mathrm{ES}=0.58$; main effect time $\mathrm{ES}=0.80$; 
condition by time interaction $\mathrm{ES}=0.15$ ). The peak in plasma $[\mathrm{TAG}]$, occurring at $5 \mathrm{~h}$ in both conditions, was lower following HIIR compared with CON (95\% CI -0.36 to -0.06 , ES = 0.70). In addition, TAUC-TAG was lower following HIIR compared with CON $(95 \%$ CI -1.18 to $-0.12, \mathrm{ES}=0.50)$ (Table 2). Differences in sub-sections of the TAUC-TAG between CON and HIIR were moderate (main effect condition 95\% CI -0.40 to $-0.04 \mathrm{ES}=0.57$ ). Specifically, TAUC-TAG was lower after HIIR compared with CON between 1 to 4.5 h (95 $\% \mathrm{CI}-0.50$ to $0.02, \mathrm{ES}=0.47)$ and 4.5 to $6.5 \mathrm{~h}(95 \% \mathrm{CI}-0.56$ to $-0.07, \mathrm{ES}=0.57)$. Individual changes (delta) in TAUC-TAG between CON and HIIR are shown in Figure 3. Ten boys responded to the interval running session (i.e., the reductions in TAUC-TAG following the high-intensity interval running exceeded the control). Percent peak heart rate during the interval runs was the only measured variable demonstrating a meaningful relationship with TAUC-TAG $(\mathrm{r}=0.65 ; 95 \% \mathrm{CI}-0.87$ to -0.20$)$, explaining $42 \%$ of the variance (Figure 4$)$. Differences in iAUC-TAG between CON and HIIR were small to moderate ( $95 \%$ CI -0.83 to $0.13, \mathrm{ES}=0.39)($ Table 2).

Postprandial plasma [glucose] did not differ meaningfully between CON and HIIR (main effect condition $95 \% \mathrm{CI}-0.30$ to 0.15 , $\mathrm{ES}=0.19$, main effect time $\mathrm{ES}=0.43$, condition by time interaction $\mathrm{ES}=0.12$ ). No meaningful difference in TAUC-glucose was evident between CON and HIIR (95\% CI -1.7 to 1.1) (Table 2). The iAUC-glucose did not differ meaningfully between CON and HIIR (95\% CI -1.76 to 1.94) (Table 2).

There was no meaningful difference in postprandial plasma [insulin] between CON and HIIR (main effect condition $95 \% \mathrm{CI}-35$ to 13 , $\mathrm{ES}=0.25$, main effect time $\mathrm{ES}=0.69$, condition by time interaction $\mathrm{ES}=0.11$ ). In addition, TAUC-insulin was similar across the experimental conditions (95\% CI -210 to 90) (Table 2). No meaningful difference in iAUCinsulin was evident between CON and HIIR (95\% CI -149 to 41) (Table 2). 


\section{DISCUSSION}

The main finding of the present study was that a single session of low-volume HIIR performed the day before standardised test meals attenuated postprandial plasma [TAG] in healthy, active 11 to 12 year old boys. To our knowledge, this is the first study to investigate the effect of HIIR on postprandial lipemia in boys. The exercise protocol was well tolerated by all participants and, therefore, may have practical applications for health in similar populations.

Changes in fasting plasma [TAG] were small to moderate following the exercise intervention, consistent with previous findings with young people involving moderate and vigorous intensity exercise $(2,39,41)$. However, fasting [TAG] is typically less predictive of cardiovascular disease risk than postprandial [TAG] (1). In addition, substantial variation is evident in fasting [TAG] in children (40), highlighting the importance of studying plasma [TAG] in the postprandial period.

Along with the plethora of studies supporting the TAG lowering effects of moderateintensity exercise in adults (34), there is growing evidence that moderate and vigorous intensity exercise promote reductions in postprandial lipemia in healthy boys $(2,29,39,41)$. The current study extends these findings by demonstrating for the first time that low volume HIIR attenuates postprandial plasma [TAG] in 11 to 12 year old boys. Several recent studies with adults also support the reduction in postprandial [TAG] following intermittent highintensity interval running (11) and all-out cycle sprints $(13,14)$. Interestingly, a small to moderate reduction in iAUC-TAG was evident after HIIR suggesting that the TAG-lowering effect of HIIR is influenced, in part, by the change in fasting [TAG] (i.e., endogenous VLDL metabolism). Nevertheless, the iAUC-TAG was lower after HIIR compared with CON 
indicating that differences in the metabolism of exogenous TAG may contribute somewhat to the lower postprandial [TAG] evident after HIIR.

Currently, the change in postprandial lipemia after exercise in young people varies, with estimated ES ranging from 0.26 to $0.77(2,29,39,41)$. However, on average, the changes are moderate and comparable to the attenuation evident after HIIR in our study. Although comparing different groups of boys indirectly may be confounded by differences in participant characteristics, it provides an important insight into the extent HIIR attenuates postprandial lipemia in this population. Previous studies with young boys demonstrate that the peak in postprandial [TAG] occurs 2 to $4 \mathrm{~h}$ following the consumption of a single standardised meal $(2,29,39,41)$. The later peak in the present study ( $\sim 5 \mathrm{~h}$ in both conditions) reflects the additional effect of the lunch meal on the postprandial lipaemic response. Differences in postprandial [TAG] between CON and HIIR emerged after $1 \mathrm{~h}$ up to the end of the postprandial period of $6.5 \mathrm{~h}$. Although other studies with boys have not examined differences in TAUC-TAG over sub-sections of the total postprandial period, the TAGlowering effect of moderate and vigorous intensity exercise appears to persist throughout the postprandial period $(2,29,39,41)$.

The effect of exercise on postprandial TAG metabolism has traditionally been linked with the exercise energy expenditure (EE) in adults (16). A recent review suggests that an energy expenditure threshold of $2 \mathrm{MJ}$ is required to elicit acute reductions in postprandial [TAG] (31). However, the evidence of a dose-dependent response in young people is not supported $(39,41)$. Although it was not possible to measure the exercise EE directly, it is reasonable to assume that the short duration of HIIR (10 minutes in total) would incur a lower EE than that reported in other studies investigating the effect of longer duration, moderate or vigorous intensity exercise on postprandial [TAG] in boys $(2,29,39,41)$, and below the $2 \mathrm{MJ}$ threshold suggested in adults (31). Estimating EE during high-intensity exercise based on 
indirect calorimetry is limited by the disturbances in the bicarbonate pool that occur during non-steady state exercise and, therefore, the gas composition of expired air is unlikely to reflect tissue metabolism (24). Indeed, Gabriel et al. (14) reported that in adults, low-volume, sprint interval cycling attenuated postprandial lipemia, and yet, no effect of brisk walking was observed on postprandial [TAG] despite a 57\% greater exercise EE estimated from the average power output and mechanical efficiency. Consequently, the capacity for highintensity exercise to reduce postprandial [TAG] suggests that the exercise intensity is important in modifying the postprandial lipaemic response.

Clear inter-individual variability is evident in the exercise-induced changes in plasma [TAG], which is consistent with previous work in boys (39) and adults (15). Average percent peak heart rate during HIIR was the only physical or exercise response variable that demonstrated a significant correlation with exercise-induced TAUC-TAG suggesting exercising at a higher relative intensity augments the attenuation in postprandial [TAG]. Although $\dot{\mathrm{VO}}_{2}$ was not measured during HIIR, neither oxygen uptake, substrate utilisation nor exercise EE have been found to contribute meaningfully to the individual heterogeneity in postprandial lipemia following moderate-intensity exercise in adults and boys previously $(15,39)$. While the majority of boys in the current study were classified as early pubescent, a range of self-assessed sexual maturity ratings were identified (pubic hair development stage 1: $n=2$; stage $2: n=11$; stage $3: n=2$ ). Subsequent analyses revealed no discernible effect of maturity status on the postprandial TAG response (data not shown). However, the influence of maturity status on postprandial lipemia is limited by the relatively small sample size, and, therefore, cannot be determined with confidence from our findings.

It is widely accepted that non-fasting TAG is an independent risk factor for future cardiovascular events $(1,33)$, and efforts to reduce cardiovascular risk factors should begin from a young age (32). However, the clinical relevance of the exercise-induced reduction in 
postprandial [TAG] cannot be determined from our findings. Currently, it has not been possible to identify a pre-defined postprandial lipaemic response in young people or adults beyond which further reductions will confer improved health. Consequently, it is not possible to identify how many participants experienced meaningful reductions in postprandial [TAG] following HIIR. Nevertheless, all participants in the present study demonstrated a healthy postprandial [TAG] profile independent of the experimental condition and the time of TAG measurement and, therefore, the potential for HIIR to elicit reductions in postprandial lipemia in individuals with normal postprandial TAG metabolism is encouraging.

The potential for low volume HIIR to reduce the postprandial lipaemic response is promising considering many young people fall short of the current physical activity recommendations (35). A perceived lack of time and enjoyment are highlighted frequently as barriers to exercise participation in adolescents (5). The total exercise time commitment (including warm-up, recovery and cool-down) in the present study was $27 \mathrm{~min}$, highlighting the time-efficiency of our HIIR session. It has been found that combining moderate-intensity exercise with periods of high-intensity effort is associated with greater perceived enjoyment than performing a similar duration of continuous, moderate-intensity exercise alone in prepubertal boys (8). Moreover, children typically spend a lower percentage of time engaged in low-intensity activities and more time on high-intensity activities compared with adults (22). Consequently, HIIR may represent an effective strategy in boys to improve health that is practical, time-efficient and enjoyable, but further research is required to support this.

In the present study, no differences in the postprandial glucose or insulin response were evident, and therefore, are unlikely to contribute to the diminished postprandial plasma [TAG] response following HIIR. The evidence for acute moderate-intensity exercise-induced changes in postprandial [glucose] following high-fat meals are generally not well supported in young people $(2,29,39,41)$. Furthermore, despite the paucity of research, no effect of 
moderate-intensity exercise was evident on the postprandial insulin profile (29). Insulin is known to play a pivotal role in TAG metabolism, regulating the uptake of TAG to skeletal muscle and adipose tissue, along with the release of very low-density lipoproteins (VLDL) from hepatic tissue. However, exercise-induced changes in postprandial lipemia appear independent of the postprandial insulin response (17).

The mechanism responsible for the acute attenuation in postprandial [TAG] following exercise is not known currently in young people and cannot be elucidated from our findings. In adults, it is proposed that enhanced removal of TAG from the blood mediated by increased lipoprotein lipase (LPL) activity in the plasma or muscle $(18,20)$ and/or a reduction in hepatic VLDL-TAG synthesis and secretion (30) is responsible. However, it is likely that enhanced muscle LPL activity is mediated by a reduction in plasma [insulin] (26), which was not observed in the present study. In support of the latter mechanism, Gill et al. (15) reported 3-hydroxybutyrate (3-OHB) concentration, a marker of hepatic fatty acid oxidation, was associated with moderate-intensity exercise-induced changes in postprandial [TAG]. However, no effect of high-intensity exercise was observed on plasma [3-OHB] in adults (14).

A possible limitation of this study is the accuracy of physical activity replication between the experimental conditions. Participants were asked to subjectively record their physical activities $48 \mathrm{~h}$ before day 2 of the first experimental condition and then replicate this during the same period before the second condition. Although this procedure was verified verbally and by comparing the diaries, in the absence of an objective measure to quantify free-living physical activity, discrepancies between the conditions would introduce variability between the conditions that may influence the postprandial measures. A further limitation concerns the fact that participants did not complete a session of similar duration moderateintensity exercise for comparison with HIIR. 
In conclusion, the present study is the first to our knowledge to show that low volume HIIR performed $\sim 15.5 \mathrm{~h}$ before a standardised breakfast reduces postprandial [TAG] in healthy, active 11 to 12 year old boys. Low volume, high-intensity exercise may be a timeefficient strategy to improve health in boys, but further work is required to examine this chronically.

\section{Acknowledgements}

The authors thank Woodbrook Vale High School in Loughborough for their support and the participants and their parents for their commitment throughout this research. We also acknowledge Josh Hill and Natalie Wheat for their assistance in data collection. The authors declare no conflict of interest. No funding was received for this research, other than that available internally through Loughborough University. The results of the present study do not constitute endorsement by the American College of Sports Medicine. 


\section{REFERENCES}

1. Bansal S, Buring JE, Rifai N, Mora S, Sacks FM, Ridker PM. Fasting compared with nonfasting triglycerides and risk of cardiovascular events in women. JAMA. 2007;298(3):309-16.

2. Barrett LA, Morris JG, Stensel DJ, Nevill ME. Exercise and postprandial plasma triacylglycerol concentrations in healthy adolescent boys. Med Sci Sports Exerc. 2007;39(1):116-22.

3. Borg GAV, Noble BJ. Perceived exertion. Exerc Sport Sci Rev. 1974;2(1):131-53.

4. Buchan DS, Ollis S, Young JD, et al. The effects of time and intensity of exercise on novel and established markers of CVD in adolescent youth. Am J Hum Biol. 2011;23(4):517-26.

5. Butt J, Weinberg RS, Breckon JD, Claytor RP. Adolescent physical activity participation and motivational determinants across gender, age, and race. $J$ Phys Act Health. 2011;8(8):1074-83.

6. Cohen J. Statistical power analysis for the behavioural sciences. 2nd ed. Hillsdale (NJ): Lawrence Erlbaum Associates; 1988. p. 23-4.

7. Cohn JS. Postprandial lipemia: emerging evidence for atherogenicity of remnant lipoproteins. Can J Cardiol. 1998;14:18B-27B.

8. Crisp NA, Fournier PA, Licari MK, Braham R, Guelfi KJ. Adding sprints to continuous exercise at the intensity that maximises fat oxidation: implications for acute energy balance and enjoyment. Metabolism. 2012;61(9):1280-8. 
9. Department of Health, Physical Activity, Health Improvement and Protection. Start active, stay active. A report on physical activity for health from the four home countries' Chief Medical Officers. London: United Kingdom Department of Health, Physical Activity, Health Improvement and Protection; 2011. 26 p. Available from UK Department of Health.

10. Dill DB, Costill DL. Calculation of percentage changes in volumes of blood, plasma, and red cells in dehydration. J Appl Physiol. 1974;37(2):247-8.

11. Ferreira AP, Ferreira CB, Souza VC, et al. The influence of intense intermittent versus moderate continuous exercise on postprandial lipemia. Clinics. 2011;66(4):535-41.

12. Field A. Discovering statistics using SPSS for windows. London: Sage Publications, 2009. p. 332, 501 .

13. Freese EC, Levine AS, Chapman DP, Hausman DB, Cureton KJ. Effects of acute sprint interval cycling and energy replacement on postprandial lipemia. J Appl Physiol. 2011;111(6):1584-9.

14. Gabriel B, Ratkevicius A, Gray P, Frenneaux MP, Gray SR. High-intensity exercise attenuates postprandial lipaemia and markers of oxidative stress. Clin Sci. $2012 ; 123(5): 313-21$

15. Gill JMR, Al-Mamari A, Ferrell WR, et al. Effect of prior moderate exercise on postprandial metabolism in men with type 2 diabetes: heterogeneity of responses. Atherosclerosis. 2007;194(1):134-43.

16. Gill JMR, Herd SL, Hardman AE. Moderate exercise and post-prandial metabolism: issues of dose-response. J Sports Sci. 2002;20(12):961-7. 
17. Gill JMR, Herd SL, Tsetsonis NV, Hardman AE. Are the reductions in triacylglycerol and insulin levels after exercise related? Clin Sci. 2002;102(2):223-31.

18. Gill JMR, Herd SL, Vora V, Hardman AE. Effects of a brisk walk on lipoprotein lipase activity and plasma triglyceride concentrations in the fasted and postprandial states. Eur J Appl Physiol. 2003;89(2):184-90.

19. Herd SL, Hardman AE, Boobis LH, Cairns CJ. The effect of 13 weeks of running training followed by $9 \mathrm{~d}$ of detraining on postprandial lipaemia. Br J Nutr. 1998;80(1):57-66.

20. Herd SL, Kiens B, Boobis LH, Hardman AE. Moderate exercise, postprandial lipemia, and skeletal muscle lipoprotein lipase activity. Metabolism. 2001;50(7):756-62.

21. Hood MS, Little JP, Tarnopolsky MA, Myslik F, Gibala MJ. Low-volume interval training improves muscle oxidative capacity in sedentary adults . Med Sci Sports Exerc. 2011;58(10):1849-56.

22. Hoos MB, Kuipers H, Gerver WJ, Westerterp KR. Physical activity pattern of children assessed by triaxial accelerometry. Eur J Clin Nutr. 2004;58(10):1425-1428.

23. Janssen I, LeBlanc AG. Systematic review of the health benefits of physical activity and fitness in school-aged children and youth. Int J Behav Nutr Phys Act. 2010;7:40.

24. Jeukendrup AE, Wallis GA. Measurement of substrate oxidation during exercise by means of gas exchange measurements. Int J Sports Med. 2005;26:S28-S37.

25. Jones AM, Doust JH. A 1\% treadmill grade most accurately reflects the energetic cost of outdoor running. J Sports Sci. 1996;14(4):321-7. 
26. Kiens B, Lithell H, Mikines KJ, Richter EA. Effects of insulin and exercise on muscle lipoprotein lipase activity in man and its relation to insulin action. J Clin Invest. $1989 ; 84(4): 1124-9$.

27. Lacour JR, Padilla-Magunacelaya S, Chatard JC, Arsac L, Barthélémy JC. Assessment of running velocity at maximal oxygen uptake. Eur J Appl Physiol. 1991;62(2):77-82.

28. Little JP, Gillen JB, Percival ME, et al. Low-volume high-intensity interval training reduces hyperglycemia and increases muscle mitochondrial capacity in patients with type 2 diabetes. $J$ Appl Physiol. 2011;111(6):1554-60.

29. MacEneaney OJ, Harrison M, O’Gorman DJ, Pankratieva EV, O’Connor PL, Moyna NM. Effect of prior exercise on postprandial lipemia and markers of inflammation and endothelial activation in normal weight and overweight adolescent boys. Eur J Appl Physiol. 2009;106(5):721-9.

30. Magkos F. Basal very low-density lipoprotein metabolism in response to exercise: mechanisms of hypotriacylglycerolemia. Prog Lipid Res. 2009;48(3-4):171-90.

31. Maraki M, Sidossis LS. Effects of energy balance on postprandial triacylglycerol metabolism. Curr Opin Clin Nutr Metab Care. 2010;13(6):608-17.

32. McGill HC, McMahan CA, Herderick EE, Malcom GT, Tracy RE, Strong JP. Origin of atherosclerosis in childhood and adolescence. Am J Clin Nutr. 2000;72(5):1307S-1315S.

33. Nordestgaard BG, Benn M, Schnohr P, Tybjærg-Hansen A. Nonfasting triglycerides and risk of myocardial infarction, ischemic heart disease, and death in men and women. JAMA. 2007;298(3):299-308. 
34. Peddie MC, Rehrer NJ, Perry TL. Physical activity and postprandial lipidemia: are energy expenditure and lipoprotein lipase activity the real modulators of the positive effect? Prog Lipid Res. 2012;51(1):11-22.

35. Riddoch CJ, Mattocks C, Deere K, et al. Objective measurement of levels and patterns of physical activity. Arch Dis Child. 2007;92(11):963-9.

36. Scarborough P, Bhatnagar P, Wickramasinghe K, Smolina K, Mitchell C, Rayner M. Coronary heart disease statistics. London: British Heart Foundation; 2010. 14 p. Available from British Heart Foundation.

37. Slaughter MH, Lohman TG, Boileau RA, et al. Skinfold equations for estimation of body fatness in children and youth. Hum Biol. 1988;60(5):709-23.

38. Tanner JM. Growth at adolescence. 2nd ed. Oxford, UK: Blackwell Scientific Publications; 1962. p. 28-39.

39. Tolfrey K, Bentley C, Goad M, Varley J, Willis S, Barrett L. Effect of energy expenditure on postprandial triacylglycerol in adolescent boys. Eur J Appl Physiol. 2012;112(1):23-31.

40. Tolfrey K, Campbell IG, Jones AM. Intra-individual variation of plasma lipids and lipoproteins in prepubescent children. Eur J Appl Physiol. 1999;79(5):449-56.

41. Tolfrey K, Doggett A, Boyd C, Pinner S, Sharples A, Barrett L. Postprandial triacylglycerol in adolescent boys: a case for moderate exercise. Med Sci Sports Exerc. 2008;40(6):1049-56.

42. Zilversmit DB. Atherogenesis: a postprandial phenomenon. Circulation. 1979;60(3):47385. 


\section{FIGURE LEGENDS}

Figure 1. Diagram of two-day study protocol. $T A G$, triacylglycerol. ${ }^{\dagger}$ Evening meal replicated from $1^{\text {st }}$ condition.

Figure 2. Fasting (F) and postprandial plasma triacylglycerol concentration [TAG] for the control condition $(\mathrm{CON})$, and the high-intensity interval running condition (HIIR) $(n=15)$. Values are mean (SD). Black rectangles denote breakfast and lunch meals consumed at 08:00 $\mathrm{h}$ and 12:00 $\mathrm{h}$ respectively. Main effect for condition $(\mathrm{ES}=$ $0.58)$; main effect for time $(\mathrm{ES}=0.80)$; condition by time interaction $(\mathrm{ES}=0.15)$.

Figure 3. Individual changes (delta) in the total area under the plasma triacylglycerol (TAG) concentration versus time curve (TAUC) between the high-intensity interval running and control conditions (HIIR minus $\mathrm{CON}, \mathrm{ES}=0.50$ ). Participant data are organised according to size of the delta change in TAUC-TAG. Negative values indicate a reduction in TAUC-TAG in the exercise condition compared with CON.

Figure 4. The relationship between individual changes (delta; Fig 3 ) in the total area under the plasma triacylglycerol (TAG) concentration versus time curve (TAUC) and average percent peak heart rate during the high-intensity interval running condition (HIIR). CON, control condition. 
Figure 1

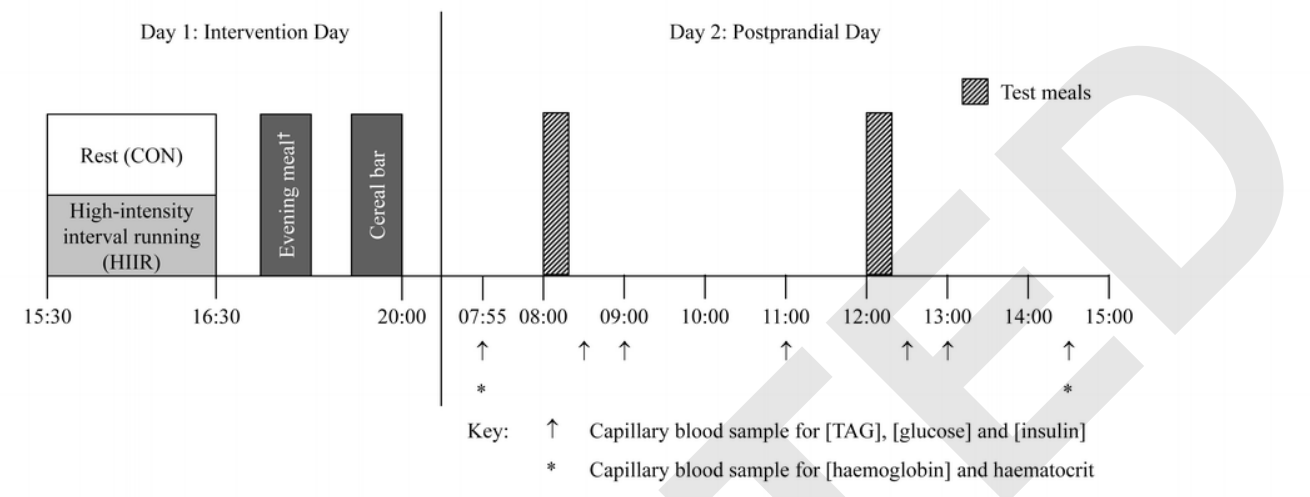


Figure 2

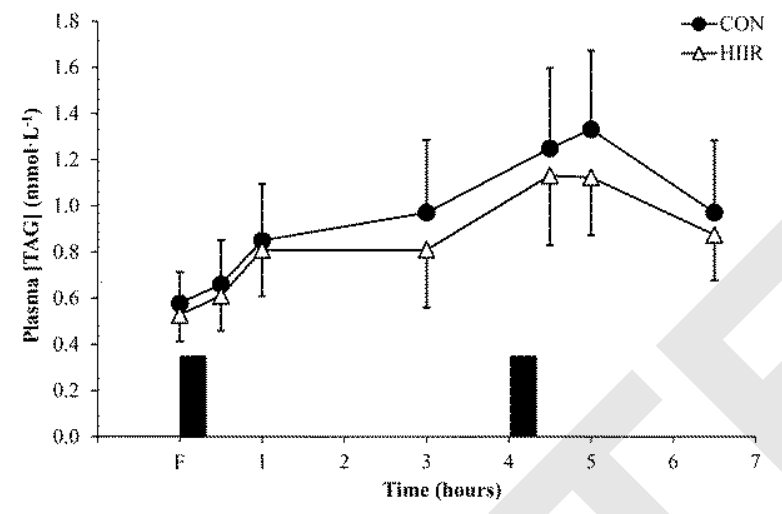


Figure 3

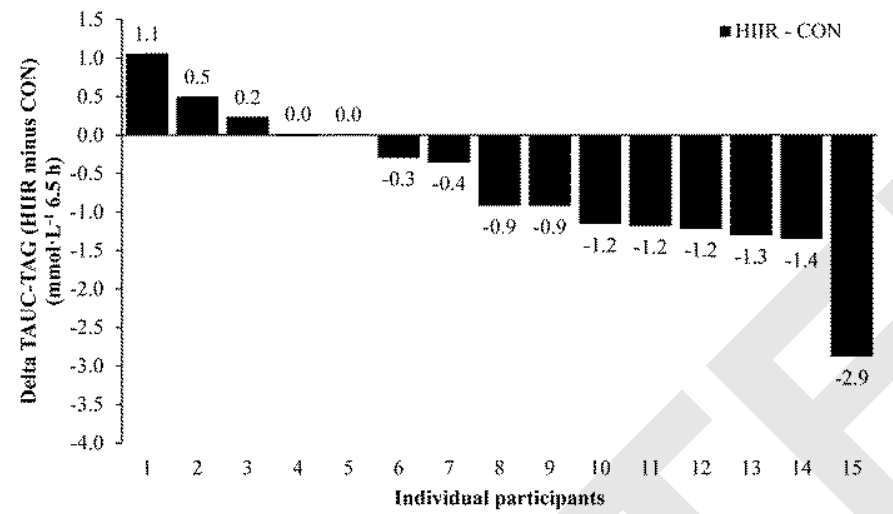


Figure 4

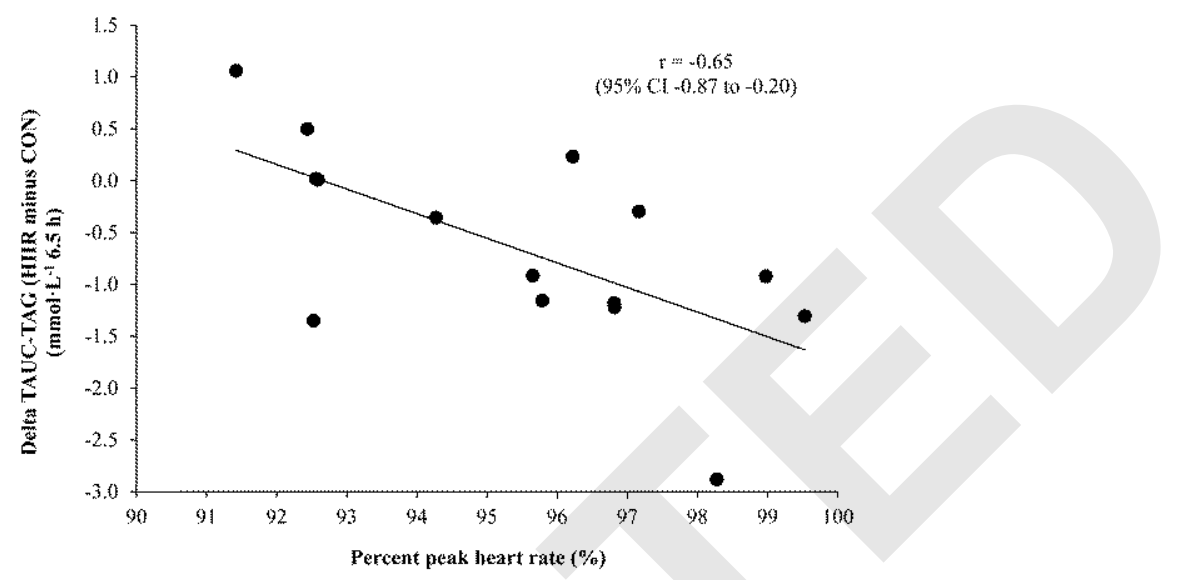


Table 1 Physical and physiological characteristics $(n=15)$

\begin{tabular}{lcc}
\hline Age (years) & 11.8 & $(0.4)$ \\
Body mass $(\mathrm{kg})$ & 42.8 & $(8.0)$ \\
Stature (m) & 1.53 & $(0.09)$ \\
Body mass index $\left(\mathrm{kg} \cdot \mathrm{m}^{-2}\right)$ & 18.3 \\
Percent body fat $(\%)$ & 13.5 \\
Lean body mass $(\mathrm{kg})$ & 36.7 \\
Genital development* & 3 \\
Pubic hair development* \\
$\quad$ VO
\end{tabular}

Values are mean (SD)

* Self assessment - median (interquartile range)

2, oxygen uptake 

the control (CON) and high-intensity interval running (HIIR) conditions $(n=15)$

\begin{tabular}{|c|c|c|c|c|c|c|c|c|c|c|c|c|}
\hline & & & & & $\mathrm{CON}$ & & HIIR & & n Difference & 95\% CI* & & |Effect size| \\
\hline $\mathrm{Fa} \mathrm{s} \quad \mathrm{t}$ & $\mathrm{i}$ & $\mathrm{n}$ & $\left.{ }^{-1}\right)$ & $\mathrm{t}$ & $0.58(\mathrm{f} .14)$ & $\mathrm{i}$ & $0.53 \mathfrak{d} 0.11)$ & & -0.05 & -0.11 to $0.0 \mathrm{~g}$ & & 0.40 \\
\hline $\mathrm{Fa} \quad \mathrm{s} \quad \mathrm{t} \quad \mathrm{i}$ & $\mathrm{i}$ & $\mathrm{n}$ & $\left.g^{-1}\right)$ & $\mathrm{g}$ & $5.52(0.33)$ & & $5.45(0 \mathrm{c} 63)$ & & $-0.07 \mathrm{~s}$ & -ce.33 to 0.19 & & 0.14 \\
\hline $\mathrm{Fa} \quad \mathrm{s} \quad \mathrm{t}$ & & $\mathrm{n}$ & $\left.g^{-1}\right)$ & $\mathrm{i}$ & 44.4 (1 7.9) & $\mathrm{s}$ & 43.4 (ํ9.9) & 1 & $-1.0 \mathrm{i}$ & $\mathrm{n}-19.0$ to 16.9 & & 0.03 \\
\hline TAUC-TAG & & ( & $\left.{ }^{-1} 16.5 \mathrm{~h}\right) \mathrm{m}$ & & o $5.83(1.416)$ & & $\cdot 5.18(1.13) \mathrm{L}$ & & -0.65 & -1.18 to -0.12 & & 0.50 \\
\hline iAUC-TA & & & $-(6.5$ him & $\mathrm{m}$ & $2.37(\Phi .03)$ & & $12.02(0.72)$ & & -0.35 & -0.83 to 0.13 & & 0.39 \\
\hline TAUC-gl u & $\mathrm{u} \quad \mathrm{c}$ & o & $\left.\mathrm{s}^{-1} 6.5 \mathrm{dl}\right)$ & & $39.8((2.6)$ & $\mathrm{m}$ & $39.5 n(1.7)$ & o & -0.31 & -1.7 to 1.1 & $\mathrm{~L}$ & 0.16 \\
\hline iAUC-gl u & $c$ & $\mathrm{o}$ & $\mathrm{s}^{-1} 6.5$ (1) & & $6.69((3.89)$ & $\mathrm{m}$ & $6.78 n(44.61)$ & o & 0.091 & -1.76 to 1.94 & $\mathrm{~L}$ & 0.02 \\
\hline TAUC-in s & $\mathrm{u}$ & 1 & $\left.\mathrm{i}^{-1} 6.5 \mathrm{nh}\right)$ & & $1272(32 \varphi)$ & & $\mathrm{m} 1212(35 \mathrm{ld})$ & & -60 & -210 to 90 & & 0.18 \\
\hline iAUC-in s & $\mathrm{u}$ & 1 & $\left.\mathrm{i}^{-1} 6.5 \mathrm{nh}\right)$ & & r004 (28p) & & m 951 (2980) & & -54 & -149 to 41 & & 0.18 \\
\hline
\end{tabular}

Values are mean (SD)

$T A G$, triacylglycerol; $T A U C$, total area under the plasma concentration versus time curve; $i A U C$, incremental area under the plasma concentration versus time curve

* 95\% confidence interval of the mean absolute difference between CON and HIIR 